\title{
Cardiopulmonary resuscitation following profound immersion hypothermia
}

\author{
David J Steedman, Timothy Rainer, Ciro Campanella
}

\begin{abstract}
A case is presented in which prolonged resuscitation and rewarming was performed following post-rescue cardiopulmonary arrest in severe immersion hypothermia. The rescue and resuscitation techniques necessary to optimise outcome in such cases are described. (f Accid Emerg Med 1997;14:170-172)
\end{abstract}

Keywords: immersion hypothermia; resuscitation

During the period of severely cold weather in the United Kingdom during the winter of 1995, the media reported on a series of tragedies from cold water immersion following falls through ice, and the heroic measures of bystanders and the emergency services to save life in these incidents. A case in which prolonged resuscitation and rewarming was performed following post-rescue cardiopulmonary arrest in severe immersion hypothermia is presented. The patient survived without neurological deficit following three and a half hours of cardiopulmonary resuscitation.

\section{Case report}

A 29 year old man fell through the ice covering of a fresh water reservoir at 14.45 hours while attempting to rescue his dog. The ambient air temperature was $-2^{\circ} \mathrm{C}$ at the time of the incident. His face did not submerge. Several bystanders were unable to reach him because the ice broke under their feet. After a hazardous operation he was eventually rescued by Fire Service personnel and transferred by stretcher to an awaiting ambulance at 16.05 hours. He was opening his eyes spontaneously and localising painful stimuli but gave no verbal response (Glasgow coma score 10/15). His pupils were equal and reactive to light. His wet clothes were carefully removed and he was wrapped in a space blanket. He was started on oxygen 6 litres/min through a face mask. The ambulance left the scene at 16.14 hours.

On route to hospital at 16.23 hours he became unresponsive and had no detectable cardiac output. The ECG monitor trace showed ventricular fibrillation. A sequence of $200 \mathrm{~J}, 200 \mathrm{~J}$, and $360 \mathrm{~J}$ dc shocks was unsuccessful. Manual external chest compres- sions with intermittent positive pressure ventilation by bag-mask was immediately started. On arrival at the accident and emergency department at 16.29 hours his clinical state was unchanged, with persisting ventricular fibrillation on the ECG monitor.

Advanced cardiopulmonary support was begun, with endotracheal intubation, intermittent positive pressure ventilation with $100 \%$ oxygen, and external chest compression using a Michigan Instrument Life Aid Resuscitator (Thumper). A rectal probe thermometer reading was $28.3^{\circ} \mathrm{C}$. A peripheral intravenous line was established and $500 \mathrm{ml}$ normal saline, warmed by a Level 1 System fluid warmer, was given.

Initial arterial blood gas measurements showed $\mathrm{H}^{+} 78.9 \mathrm{nmol} / \mathrm{l}, \mathrm{PCO}_{2} 1.56 \mathrm{kPa}$, bicarbonate $3.7 \mathrm{mmol} / 1$, and $\mathrm{Po}_{2} 77.3 \mathrm{kPa}$. His urea concentration was $8.9 \mathrm{mmol} / \mathrm{l}$, creatinine $232 \mu \mathrm{mol} / \mathrm{l}$, sodium $146 \mathrm{mmol} / 1$, potassium 6.1 $\mathrm{mmol} / \mathrm{l}$, and glucose $6.7 \mathrm{mmol} / \mathrm{l}$. A nasogastric and a urinary catheter were passed. Two peritoneal dialysis catheters and two left chest tubes were inserted and continuous pleural and peritoneal lavage performed with warmed saline. His rectal temperature had risen to $29.1^{\circ} \mathrm{C}$ at 17.00 hours. He remained in ventricular fibrillation resistant to further $d c$ shocks.

$\mathrm{He}$ was transferred to the cardiothoracic theatre at 17.25 hours. Cardiopulmonary bypass was established at 18.46 hours; one hour and 15 minutes were spent in theatre trying to cannulate the femoral vessels in the hope of being able to establish cardiopulmonary bypass without opening the chest and therefore disconnecting the patient from the thumper. It was assessed that the thumper was producing a pulsatile flow easily palpable at the femoral site. Cannulation proved extremely difficult due to a combination of vasospasm and small sized femoral artery and vein. The attempt was abandoned and attention concentrated on the chest. The total time between stopping the thumper and placing the patient on cardiopulmonary bypass using aortic and right atrial cannulation was two minutes and 45 seconds. During that time, the heart was internally massaged by the first surgeon while the assistant was placing the purse string sutures onto the ascending aorta and right atrium and 
proceeding towards cannulation. In half an hour of full flow, the temperature was raised from $25^{\circ} \mathrm{C}$ to $31^{\circ} \mathrm{C}$. At 19.00 hours, three internal dc shocks at $20 \mathrm{~J}, 20 \mathrm{~J}$, and $40 \mathrm{~J}$ were delivered, with no response. At 19.12 hours defibrillation was successful after one further $20 \mathrm{~J} \mathrm{dc}$ shock, when core temperature had risen to $37.0^{\circ} \mathrm{C}$. The ECG monitor showed sinus tachycardia with frequent ventricular ectopic beats. He was disconnected from bypass at 19.54 hours. He was transferred from theatre to the intensive care unit at 21.35 hours on inotropic support. In intensive care he was ventilated for 72 hours, requiring infusions of adrenaline, noradrenaline, dopamine, and lignocaine.

\section{Discussion}

Immersion in cold water can cool the core body temperature much more rapidly than exposure to cold air because the thermal conductivity of water is 25 times greater than that of air. The cooler the water the faster the cooling rate. ${ }^{1}$ As core body temperature declines, the basal metabolic rate and oxygen consumption fall gradually but progressively. There is a linear decrease in cerebral metabolism of $6-7 \%$ per ${ }^{\circ} \mathrm{C}$ between core temperatures of $35^{\circ} \mathrm{C}$ to $25^{\circ} \mathrm{C}^{2}$ Immersion hypothermia provides substantial protection to the central nervous system from hypoxia. With a significant drop in core temperature the immersion hypothermic casualty may appear clinically dead (without palpable pulse, blood pressure, or respiration), but may still be successfully resuscitated with little or no neurological deficit. Full recovery has occurred in a patient with an initial core temperature of $15.2^{\circ} \mathrm{C}^{3}$ The only certain diagnosis of death is failure to recover on rewarming. The diagnosis of death should be made in hospital unless conditions render prolonged attempts at resuscitation or evacuation impossible.

Hypothermic casualties, as in the case illustrated, are at risk of death even though they are alive when rescued. ${ }^{4}$ After the casualty has been removed from cold water the core temperature may continue to fall, even paradoxically during the early stages of rewarming. This phenomenon of "after drop" is caused by the continued conduction of heat down a thermal gradient from the relatively warm core to the colder peripheral shell. ${ }^{5}$ As the heart cools it becomes more susceptible to mechanical irritation which may trigger ventricular fibrillation. ${ }^{4}$ Postulated mechanisms for hypothermia induced cardiac irritability include re-entry conduction producing circus movement, and the development of independent electrical foci. ${ }^{6}$

Cold water immersion may lead to loss of the normal cardiovascular reflexes which, together with the sudden reduction in the hydrostatic squeeze to tissues below the water surface, may precipitate severe hypotension on removal from the water. ${ }^{7}$ Rescuers should attempt to retrieve the casualty horizontally from the water. ${ }^{8}$ If the patient cannot be removed horizontally, this position should be achieved as quickly as possible after removal from the cold water. Conscious patients should not be required to assist in their own rescue, as physical activity increases the after drop in core temperature by increasing perfusion of cold muscle with relatively warm blood, and also increases demands on the cold ischaemic heart. ${ }^{4}$

Following rescue further heat loss should be prevented by insulating the body surface. Until the casualty is indoors, wet clothes should not be removed, but layers of insulating material should be put on top of the clothing and covered with a layer that is water and windproof ${ }^{8}$; the head must be included, and insulation should be provided between the body and the ground. ${ }^{9}$ Space blankets, although often recommended, are no better than a similar thickness of polythene. ${ }^{4}$ Once the victim is in warm shelter and out of the wind, wet clothing should be removed if sufficient rescuers are available to do this with minimal movement of the casualty.

Palpation of peripheral pulses is difficult in hypothermic vasoconstricted patients when the cardiac rate is extremely slow. The carotid or femoral pulse should be careful palpated for at least one minute. ${ }^{10}$ The low cardiac output may be sufficient to meet the metabolic demands in these patients and ventricular fibrillation can be precipitated by unnecessary external chest compressions. In hypothermic victims chest compression should be performed at the same rate as in normothermic patients, despite changes in the compressibility of the heart and compliance of the chest wall. ${ }^{11}$

Oxygen should be given to profound hypothermic casualties when available. Care should be taken not to leave the oxygen cylinder lying on cold ground or in snow. Changing a patient from warm expired air resuscitation to assisted ventilation with oxygen from a cold cylinder can precipitate ventricular fibrillation. Tracheal intubation should be performed gently and preoxygenation will decrease the risk of precipitating ventricular fibrillation. ${ }^{12}$ Intravenous access may be extremely difficult to achieve in the field because of the intense peripheral vasoconstriction. Most drugs are ineffective in hypothermic patients or are dangerous to use because of increased cardiac irritability. ${ }^{13}$ Toxic levels may develop since drug metabolism by the hypothermic liver and kidneys is reduced. ${ }^{14}$

If ventricular fibrillation is detected, emergency personnel should deliver three shocks to determine fibrillation responsiveness. ${ }^{12}$ If ventricular fibrillation persists after three shocks, further shocks should be avoided. Attempts at defibrillation are usually unsuccessful until the core temperature is above $30^{\circ} \mathrm{C}$. Prolonged field treatment should be avoided.

There are three techniques of rewarming: spontaneous, active external, and active internal. ${ }^{15}$ Spontaneous rewarming is achieved by insulating the patient with an appropriate material, thereby preventing further heat loss and allowing the body to rewarm through endogenously generated metabolic heat. Active rewarming is indicated when endogenous thermogenesis is insufficient to produce an 
adequate rate of rewarming following insulation. Active external rewarming using a hot bath, plumbed garments, hot water bottles, and heat pads and blankets applied to the trunk have been advocated in victims of immersion hypothermia with a palpable pulse. Active rewarming is usually required when the core temperature is below $32^{\circ} \mathrm{C}$. Cardiovascular collapse associated with sudden peripheral vasodilatation is a potential complication of these techniques, and careful monitoring is essential. Resuscitation is impossible in a hot water bath

Patients in cardiorespiratory arrest require rapid temperature elevation using active internal rewarming techniques. Options include peritoneal and pleural lavage ${ }^{16}$ heated humidified oxygen, ${ }^{17}$ and mediastinal irrigation and direct myocardial lavage. ${ }^{18}$ Intravenous fluids should be warmed; however, the risk of fluid overload precipitating pulmonary oedema limits their use as a means of rewarming. The use of Ringer's lactate solution should be avoided because hepatic metabolism of lactate is reduced in hypothermia. Administration of heated fluids through a central venous cannula can produce myocardial thermal gradients and precipitate arrhythmias.

Extracorporeal circulation is the treatment of choice in hypothermic cardiac arrest. ${ }^{12141519}$ The main advantage of rewarming by cardiopulmonary bypass is the preservation of blood flow to the brain and other vital organs and rapid core temperature elevation of $1-2^{\circ} \mathrm{C}$ every three to five minutes. However, cardiopulmonary bypass can only be performed by an experienced team and when the equipment is available. Consequently cardiopulmonary bypass in practice is usually instituted after other active internal rewarming methods have been started.

The longest reported conventional cardiopulmonary resuscitation in a hypothermic victim followed by survival is six hours $30 \mathrm{~min}$ utes. ${ }^{20}$ Although the aphorism "No one is dead until warm and dead" is generally applicable, rewarming to near normal core temperature is not practicable in every case. Rewarming efforts should probably be continued until the core temperature is at least $32^{\circ} \mathrm{C}$ and may be discontinued if the patient continues to show no effective cardiac rhythm and remains totally unresponsive to all treatments. ${ }^{12}$ However, the decision to terminate resuscitation must take into account the circumstances of each incident.

Resuscitation of the patient was achieved through a joint effort, and the participation of physicians, nurses, technicians, and members of the emergency services who assisted is gratefully acknowledged.

1 Hayward JS, Eckerson JD, Collis ML. Thermal balance and survival time prediction of man in cold water. Can J Physio survival time prediction of man

2 Reuler JB. Hypothermia: pathophysiology, clinical settings and management. Ann Intern Med 1978;89:519-27.

3 Nozaki $R$, Ishibashi $K$, Adachi $N$, Nishihara $S$, Adachi $S$. Accidental profound hypothermia. N Engl J Med 1986; 315:1680.

4 Lloyd EL. Hypothermia and cold. Sci Prog Oxf 1989;73: 101-16.

5 Webb P. After drop of body temperature during rewarming: an alternative explanation. J Appl Physiol 1986;60:385-90.

6 Southwick FS, Dalgleish PH. Recovery after prolonged asystolic cardiac arrest in profound hypothermia. JAMA 1980;243:1250-3.

7 Golden FStC. Problems of immersion. Br J Hosp Med 1980;24:371-4

8 Medical commission on accident prevention. Report of the working party on out of hospital management of hypotherworking party on out of hospital management of hypother-

9 Lloyd EL. Temperature and performance. 1. Cold. BMJ 1994;309:531-4.

10 Harries M. Near drowning: In: Colquhoun ML, Handley AJ, Evans JR, eds. $A B C$ of resuscitation. London: BMJ publishing group, 1995:50-3.

11 Steedman DJ. Environmental medical emergencies. Oxford Oxford University Press, 1994:1-25.

12 Weinberg AD. Hypothermia. Ann Emerg Med 1993;22 370-7.

13 Keatinge WR. Hypothermia: dead or alive? BMJ 1991;302:

14 Danzl DF, Pozos RS. Accidental hypothermia. N Engl J Med 1994;331:1756-60.

15 Larach MG. Accidental hypothermia. Lancet 1995;345: 493-8.

16 Otto RJ, Metzler MH. Rewarming from experimental hypothermia: comparison of heated aerosol inhalation peritoneal lavage and pleural lavage. Crit Care Med 1988; $16: 869-75$

17 Lloyd EL. Equipment for airway warming in the treatmen of accidental hypothermia. J Wilderness Med 1991;2:330 50.

18 Brunette DD, Biros M, Mlinek EJ, Erlandson C, Ruiz E. Internal cardiac massage and mediastinal irrigation in hypothermic cardiac arrest. Am J Emerg Med 1992;10: 32-4.

19 Walpoth BH, Locher T, Leupi F, Schupbach P, Muhlemann W, Althaus U. Accidental deep hypothermia with cardiopulmonary arrest: extracorporeal blood rewarming in 11 patients. Eur J Cardiothorac Surg 1990;4:390-3.

20 patients. Eur J Cardiothorac Surg 1990;4:390-3. Lexow $K$. Severe accidental hypothermia. survival after Med Res 1991;50(suppl 6):112-4.
Department of

Emergency Medicine,

St George Hospital,

Kogarah, NSW 2217, Australia

G J Hollis

Correspondence to:

Dr G J Hollis, Registrar,

Department of Emergency

Medicine.

Accepted for publication 6 February 1997

\section{Sixth cranial nerve palsy following closed head injury in a child}

\author{
Abstract \\ A five year old female had an isolated \\ abducens nerve palsy following closed \\ head injury. There was no associated skull \\ fracture, haematoma, or other cranial \\ nerve injury. The significance, frequency,
}

and differential diagnosis of traumatic sixth cranial nerve injury is discussed, particularly in paediatric patients. Management is symptomatic; occlusion with an eye pad may be used if diplopia is significant. In young children alternate 